Canad. Math. Bull. Vol. 21 (1), 1978

\title{
ON INTERTWINING AND FACTORIZATION BY SELF-ADJOINT OPERATORS
}

\author{
BY \\ C.-S. LIN AND M. RADJABALIPOUR
}

\begin{abstract}
In this paper we first study the equality of two operators whose values at each point satisfy certain inequalities, and then, somehow related, we examine the possibility of writing certain operators as products of two self-adjoint operators.
\end{abstract}

By an operator we mean a bounded linear transformation defined on a (finite or infinite-dimensional) complex Hilbert space $H$. An operator $W$ is called a quasiaffinity if it is injective and has a dense range. An operator $S$ is said to be a quasiaffine transform of an operator $T$ if $T W=W S$ for some quasiaffinity $W$.

Let $S$ and $T$ be positive operators, then $\|S x\|=\|T x\|$ for all $x \in H$ if and only if $S=T$. However, the simple counterexample

$$
S=\left[\begin{array}{ll}
1 & 0 \\
0 & 1
\end{array}\right], \quad T=\left[\begin{array}{rr}
1 & 0 \\
0 & -1
\end{array}\right]
$$

on $\mathbb{C}^{2}$ shows that if $S$ and $T$ are not positive, then, although $\|S x\|=\|T x\|=$ $\left\|S^{*} x\right\|=\left\|T^{*} x\right\|$ for all $x \in H, S$ and $T$ are not even similar. In the following, as a corollary to a more general theorem, we show that for any operators $S$ and $T$ if $\|S x\| \leq\|T x\|$ and $\left\|T^{*} x\right\| \leq\left\|S^{*} x\right\|$ for all $x \in H$, and if $S$ is a quasiaffine transform of $T$, then $S$ and $T$ are unitarily equivalent; in fact, if $W S=T W$ for a quasiaffinity $W$ and if $P U$ is the polar decomposition of $W$, then $T=U S U^{*}$.

Later on as we study the products of self-adjoint operators we also provide some examples related to this result.

THEOREM 1. Let $S_{1}, S_{2}, T_{1}, T_{2}, W_{1}$ and $W_{2}$ be operators satisfying the following conditions:

(1) $\left\|S_{1} x\right\| \leq\left\|T_{1} x\right\|$ for all $x \in H$;

(2) $\left\|T_{1}^{*} x\right\| \leq\left\|S_{1}^{*} x\right\|$ for all $x \in H$;

(3) $\left\|S_{2} x\right\| \leq\left\|T_{2} x\right\|$ for all $x \in H$;

(4) $\left\|T_{2}^{*} x\right\| \leq\left\|S_{2}^{*} x\right\|$ for all $x \in H$;

(5) $W_{1}$ and $W_{2}$ have finite-dimensional null spaces, and $T_{1} W_{2}=W_{1} S_{2}$ and $T_{2} W_{2}^{*}=W_{1}^{*} S_{1}$.

Then the inequalities (3) and (4) are actually equalities. Moreover, if $W_{1}$ and $W_{2}$ are quasiaffinities with polar decompositions $W_{i}=P_{i} U_{i}(i=1,2)$, then (1)-(4) become equalities, and $T_{1}=U_{1} S_{2} U_{2}^{*}$ and $S_{1}=U_{1} T_{2} U_{2}^{*}$.

Received by the editors November 23, 1976 and, in revised form, May 17, 1977. 
Proof. It is easy to see that the operators

$$
M_{1}=\left[\begin{array}{cc}
0 & T_{1} \\
S_{1}^{*} & 0
\end{array}\right] \text { and } M_{2}=\left[\begin{array}{cc}
0 & T_{2} \\
S_{2}^{*} & 0
\end{array}\right]
$$

on $H \oplus H$ are hyponormal, and $M_{1} B=B M_{2}^{*}$, where

$$
B=\left[\begin{array}{cc}
W_{1} & 0 \\
0 & W_{2}
\end{array}\right]
$$

(Recall that an operator $A$ is called hyponormal if $\left\|A^{*} x\right\| \leq\|A x\|$ for all $x \in H$ ). Thus $M_{2}$ is a normal operator by Corollary 2 of [3], and hence $\left\|T_{2} x\right\|=\left\|S_{2} x\right\|$ and $\left\|S_{2}^{*} x\right\|=\left\|T_{2}^{*} x\right\|$ for all $x \in H$.

Now, assume $W_{1}$ and $W_{2}$ are quasiaffinities with the aforementioned polar decompositions. By [3, Theorem 3 (a)] both $M_{1}$ and $M_{2}$ are normal and therefore (1) and (2) also become equalities. Moreover, $M_{1} P U=P U M_{2}$, where $P=P_{1} \oplus P_{2}$ and $U=U_{1} \oplus U_{2}$. Thus, $M_{1} P=P U M_{2} U^{*}$. Since $P$ is positive and injective, the Putnam-Fuglede theorem (see [2, page 99]) implies that

$$
\left[\begin{array}{cc}
0 & T_{1} \\
S_{1}^{*} & 0
\end{array}\right]=\left[\begin{array}{cc}
0 & U_{1} S_{2} U_{2}^{*} \\
U_{2} T_{2}^{*} U_{1}^{*} & 0
\end{array}\right] .
$$

Thus, $T_{1}=U_{1} S_{2} U_{2}^{*}$ and $S_{1}=U_{1} T_{2} U_{2}^{*}$.

Corollary. Let $S$ and $T$ be operators. If $\|S x\| \leq\|T x\|$ and $\left\|T^{*} x\right\| \leq\left\|S^{*} x\right\|$ for all $x \in H$, and if $W S=T W$ and $W^{*} S=T W^{*}$ for some quasiaffinity $W$, then $T$ and $S$ are unitarily equivalent. In particular, if $W$ is an injective positive operator, then $S=T$.

Proof. Let $S=S_{1}=S_{2}, T=T_{1}=T_{2}$ and $W=W_{1}=W_{2}$ in Theorem 1 , then the first part follows. If $W$ is injective and positive, so is $B$ in the proof of Theorem 1. Since $M_{1} B=B M_{2}^{*}$, and $M_{1}$ and $M_{2}^{*}$ are normal, $M_{1}=M_{2}^{*}$ by the Putnam-Fuglede theorem. Hence $S=T$.

Remark 1. As it is shown in [7, Example 1], if $T$ is the bilateral shift $T e_{n}=e_{n+1}$ on some basis $\left\{e_{n}\right\}_{-\infty}^{\infty}$ of $H$, and if $S=T^{*}$, then $\|S x\|=\|T x\|=\left\|T^{*} x\right\|=$ $\left\|S^{*} x\right\|$ for all $x \in H$ and $W S=T W$, where $W$ is the symmetry (i.e., the selfadjoint unitary operator) defined by $W e_{n}=e_{-n}(n=0, \pm 1, \pm 2, \ldots)$. However, $S \neq T$. Thus, positivity of $W$ in above corollary is essential.

Remark 2. Let $W$ and $X$ be two non-commuting positive, injective operators. Let $T=W X$ and $S=T^{*}$. Then $W S=T W$ and $S \neq T$. Therefore, according to Corollary above, at least one of the two inequalities $S^{*} S \leq T^{*} T$ and $T T^{*} \leq S S^{*}$ does not hold. However, the following theorem shows that none of these can hold. Note that the truth of any of these inequalities implies that either $T$ or $T^{*}$ is hyponormal. This theorem and the consequent ones are independently interesting and study certain operators in $\mathcal{M}^{2}$, where $\mathcal{M}$ denotes 
the class of all self-adjoint operators and

$$
\mathcal{M}^{2}=\{A B: A \in \mathcal{M} \quad \text { and } B \in \mathcal{M}\}
$$

(The latter class is defined in [4] and some of its properties are studied there).

An operator $T$ is called dominant if for every $z \in \mathbb{C}$ there exists a positive number $M_{z}$ such that $\left\|\left(\bar{z}-T^{*}\right) x\right\| \leq M_{z}\|(z-T) x\|$ for all $x \in H$; if the numbers $M_{z}$ are all less than a positive number $M$, we say that $T$ is an $M$-hyponormal operator. Note that $T$ is hyponormal if and only if it is 1-hyponormal.

TheOREM 2. An M-hyponormal operator $T \in \mathcal{M}^{2}$ is necessarily normal.

Proof. Let $T=A B$ with $A=A^{*}$ and $B=B^{*}$. Consider two cases;

CASE 1. $A$ is injective. Then $A T^{*}=T A$ and thus, by [3, Theorem 3 (a)], $T$ is a normal operator.

Case 2. A has a nontrivial null space $N$. Let

$$
A=\left[\begin{array}{cc}
A_{1} & 0 \\
0 & 0
\end{array}\right] \text { and } B=\left[\begin{array}{ll}
B_{1} & B_{2} \\
B_{3} & B_{4}
\end{array}\right]
$$

with respect to $N^{\perp} \oplus N$, where $A_{1}$ is an injective self-adjoint operator and $B_{1}$ is self-adjoint. Then

$$
T=\left[\begin{array}{cc}
A_{1} B_{1} & A_{1} B_{2} \\
0 & 0
\end{array}\right] .
$$

Since $A_{1} B_{1}$ is the restriction of $T$ to an invariant subspace, $A_{1} B_{1}$ is $M$ hyponormal and thus in view of Case 1 it is normal. Hence by [3, Theorem 4], $N$ is a reducing invariant subspace of $T$ and therefore $A_{1} B_{2}=0$. This shows that $T$ is a normal operator.

\section{Corollary. A hyponormal (subnormal) operator in $\mathcal{M}^{2}$ is normal.}

Remark 3. Halmos [2, page 270] shows that the unilateral shift is not the product of a finite number of self-adjoint operators. The proof there much depends on the left-invertibility of the unilateral shift and does not have a generalization to non-normal hyponormal operators. Our Corollary shows that this generalization is true if we restrict ourselves to only two self-adjoint operators. Our proof also much depends on the fact that only two self-adjoint operators are present and does not have a generalization to an arbitrary number.

We are grateful to the referee who pointed out that any unitary is the product of four symmetries (see [2], page 269). Hence any invertible hyponormal operator is in $M^{5}$.

For dominant operators we have the following theorem. 
TheOREM 3. Let $T$ be a dominant operator, and assume that $T=A B$, where $A \geq 0$ and $B=B^{*}$. Then $T$ is a normal operator.

Proof. Write

$$
A=\left[\begin{array}{ll}
0 & 0 \\
0 & C
\end{array}\right] \text { and } B=\left[\begin{array}{ll}
B_{1} & B_{2} \\
B_{3} & B_{4}
\end{array}\right]
$$

where $C$ is an injective positive operator.

Since

$$
T=A B=\left[\begin{array}{cc}
0 & 0 \\
C B_{3} & C B_{4}
\end{array}\right]
$$

it follows that $\mathrm{CB}_{4}$ is also dominant. But

$$
\left(C B_{4}\right) C^{1 / 2}=C^{1 / 2}\left(C^{1 / 2} B_{4} C^{1 / 2}\right),
$$

which implies that $C B_{4}$ is a normal operator [6, Theorem 1]. (Note that $C^{1 / 2} B_{4} C^{1 / 2}$ is self-adjoint). Now, by [3, Theorem 4], $C B_{3}=0$ and hence $T$ is normal.

For quasinilpotent operators the following theorem is valid.

THEOREM 4. A quasinilpotent operator $T$ is of the form $T=A B$ with $A \geq 0$ and $B=B^{*}$ if and only if $T^{2}=0$.

Proof. Assume that $T=A B$ with $A \geq 0$ and $B=B^{*}$. As in the proof of Theorem 3, write

$$
A=\left[\begin{array}{ll}
0 & 0 \\
0 & C
\end{array}\right], \quad B=\left[\begin{array}{ll}
B_{1} & B_{2} \\
B_{3} & B_{4}
\end{array}\right] \text { and } A B=\left[\begin{array}{cc}
0 & 0 \\
C B_{3} & C B_{4}
\end{array}\right],
$$

where $C$ is a positive and injective operator and $C B_{4}$ is a quasinilpotent operator. Since $\left(C B_{4}\right) C^{1 / 2}=C^{1 / 2}\left(C^{1 / 2} B_{4} C^{1 / 2}\right)$, by [1, Theorem 3], $C B_{4}$ is similar to the self-adjoint operator $C^{1 / 2} B_{4} C^{1 / 2}$ and hence $C B_{4}=0$. Now, it is easy to see that $(A B)^{2}=0$.

Conversely, assume that $T^{2}=0$. Suppose that $T \neq 0$, otherwise the proof will be trivial. Since the range of $T$ is included in the null space of $T$, it follows that $T$ has the form

$$
T=\left[\begin{array}{ll}
0 & D \\
0 & 0
\end{array}\right]
$$

Let

$$
A=\left[\begin{array}{ll}
I & 0 \\
0 & 0
\end{array}\right] \text { and } B=\left[\begin{array}{cc}
0 & D \\
D^{*} & 0
\end{array}\right]
$$

Obviously, $A \geq 0, B=B^{*}$ and $T=A B$. 
The following example shows that the positivity of one of the factors $A$ and $B$ in Theorem 4 is essential.

EXAMPLE. Let

$$
T=\left[\begin{array}{lll}
0 & 1 & 0 \\
0 & 0 & 1 \\
0 & 0 & 0
\end{array}\right], \quad A=\left[\begin{array}{lll}
0 & 0 & 1 \\
0 & 1 & 0 \\
1 & 0 & 0
\end{array}\right], \quad B=\left[\begin{array}{lll}
0 & 0 & 0 \\
0 & 0 & 1 \\
0 & 1 & 0
\end{array}\right] .
$$

It is easy to see that $T^{3}=0, T^{2} \neq 0, A=A^{*}, B=B^{*}$ and $T=A B$. In fact, in a finite-dimensional Hilbert space, since every nilpotent operator is similar to its adjoint, it follows from [5, Theorem 1] that every nilpotent operator is in $\mathcal{M}^{2}$.

Added in proof. The following example shows that there exists a nonnormal hyponormal operator in $\mathcal{M}^{3}$ (cf. Theorem 2). Let $H$ be a Hilbert space with a (bilateral) basis $\left\{e_{n}\right\}_{-\infty}^{\infty}$. Let $U$ be the bilateral shift defined by $U e_{n}=e_{n+1}$. Let $P$ be the diagonal operator $P e_{n}=\alpha_{n} e_{n+1}$, where $\left\{\alpha_{n}\right\}_{-\infty}^{\infty}$ is an increasing (bilateral) sequence of positive numbers. Then $P U$ is a non-normal hyponormal operator in $\mathcal{M}^{3}$ (see [2, pages 269 and 312]).

\section{REFERENCES}

1. C. K. Fong and M. Radjabalipour, On quasiaffine transforms of spectral operators, Michigan Math. J. 23 (1976), 147-150.

2. P. R. Halmos, A Hilbert Space Problem Book, Van Nostrand, Princeton, 1967.

3. M. Radjabalipour, On majorization and normality of operators, Proc. Amer. Math. Soc., 62 (1977), 105-110.

4. H. Radjavi, On self-adjoint factorization of operators, Can. J. Math. 21 (1969), 1421-1426.

5. H. Radjavi and J. P. Williams, Product of self-adjoint operators, Michigan Math. J. 16 (1969), $177-185$.

6. J. G. Stampfli and B. L. Wadhwa, An asymmetric Putnam-Fuglede theorem for dominant operators, Ind. Univ. Math. J. 25 (1976), 359-365.

7. J. P. Williams, Operators similar to their adjoints, Proc. Amer. Math. Soc., 20 (1969), 121-123.

St. Thomas UnIVERSITY,

Fredericton, N. B., CANADA.

and

REZA SHAH KabIR UNIVERSITY, IRAN. 